\title{
Research on Information Sharing between the Hospital and the Community based on the Regional Health Information Platform
}

\author{
Jing $\mathrm{Li}^{\mathrm{a}}$, Hong Zhang ${ }^{\mathrm{b}, *}$ and Yan Bai ${ }^{\mathrm{c}}$ \\ Computer center of Guang, anmeng Hospital, China Academy of Chinese Medical Science, Beijing \\ 100053, China \\ a284279676@qq.com, b'zhanghong6699@163.com, 'c11025314@qq.com
}

Keywords: Regional Health Information Platform; Hospital; Community; Information Sharing.

\begin{abstract}
With the deepening development of the medical and health reform and the rapid development of information technology in the medical and health industry, by the methods of information technology to innovate the services of medical and health has got widespread concern of the public. Combing with the demand of the information sharing between the hospital and the community, the paper first analyses the present situation and the existing problems and then proposes the sharing mode between the hospital and the community based the regional health information platform. Secondly the paper introduces the overall framework of the regional health information platform and the interaction process between the hospital and the community based the regional health information platform. Then the paper analyses the key technology of the information sharing between the hospital and the community based on the regional health information platform, including the information standardization, data acquisition and access technology, network transmission technology, data storage technology and information security technology. The information sharing between the hospital and the community based on the regional health information platform improves the level of the diagnosis and treatment in the community and provides the technical support for the implementation of the grading treatment mechanism.
\end{abstract}

\section{Introduction}

With the continuous development of the hospital information most hospitals have established hospital information systems with electronic medical record as the core that has realized interconnections and information exchanges between various information systems in the hospital to meet the internal use of the medical resource. But the information systems in different hospitals or information systems between the hospital and the community are isolated. For patients the most convenient choice is to be treated in the community. But if there are the situations that the patients get the difficult miscellaneous diseases or cannot be diagnosed, the patients need to be transferred to the higher level hospital. The communities in Beijing have basically established information systems which provide the function of basic medical services, health records management and chronic diseases management etc [1].

If the sharing and exchange of information between the hospital and the community can be realized, the doctors in the hospital can see the information of electronic health records and the slow disease management recorded in the community for the patients that will provide great help for the diagnosis of doctors. In addition, if the treatment information of patients in the hospital including inspection information, image information and the electronic medical records can be accessed to view through the information technology by the doctors in the community that will make patients enjoy the diagnosis of the large hospital in the community and also will improve the level of the medical service of the community. Exploring and constructing the information sharing platform oriented the Regional Medical Union which is the import work of the "Wisdom Beijing " project in 2014 will provide technical support for the mechanism of the first diagnosis in the community, grading treatment and the two way referral between the community and the hospital. Therefore the paper analysis the present situation of the information sharing and the information exchange between the 
hospital and the community, then proposes the information sharing mode based the regional information platform and finally studies the key technologies.

\section{Present Situation and Analysis of Existing Problems}

Hospital information systems are established under the unified planning of the hospital and start early in order to meet the need of clinical applications. Community health information systems are unified deployed by the Health Bureau in order to meet the application of daily diagnosis and health service management in the community. The two systems are derived from different manufacturers and the development standards are not unified. Therefore to achieve the information sharing and exchange between the hospital information system and the community information system need by the way of information sharing and exchange platform.

Traditional information sharing and exchange platform is established by the hospital to meet the information interaction with the community of the hospital. That way only meets the information sharing and exchange between individual hospitals and their communities.The information cannot be achieved between the other hospitals and communities. With the development of regional medical information sharing and exchange the construction of the regional health information platform is the inevitable trend of the development for the medical information technology in order to realize the information sharing and interconnection in different institutions.

\section{Overall Framework}

The overall framework of the regional health information platform. The overall framework of the regional health information platform is shown in Fig.1.Firstly the regional health information platform builds the electronic health record data set and data set of the medical and health management by the unified information standard and information specification. Secondly the regional health information platform interconnects between different information systems in the region by the way of the information technology including the hospital information system, the community information system, the disease control system and the maternal and child health care information system, etc. The regional health information platform realizes the information interoperability and sharing between the medical institutions at all levels and units in the region which promotes the regional ability of the emergency command, decision making and the public health service [2]. The regional health information platform will finally solve the problem of the difficulty of getting medical service by the reasonable distribution of medical resources.

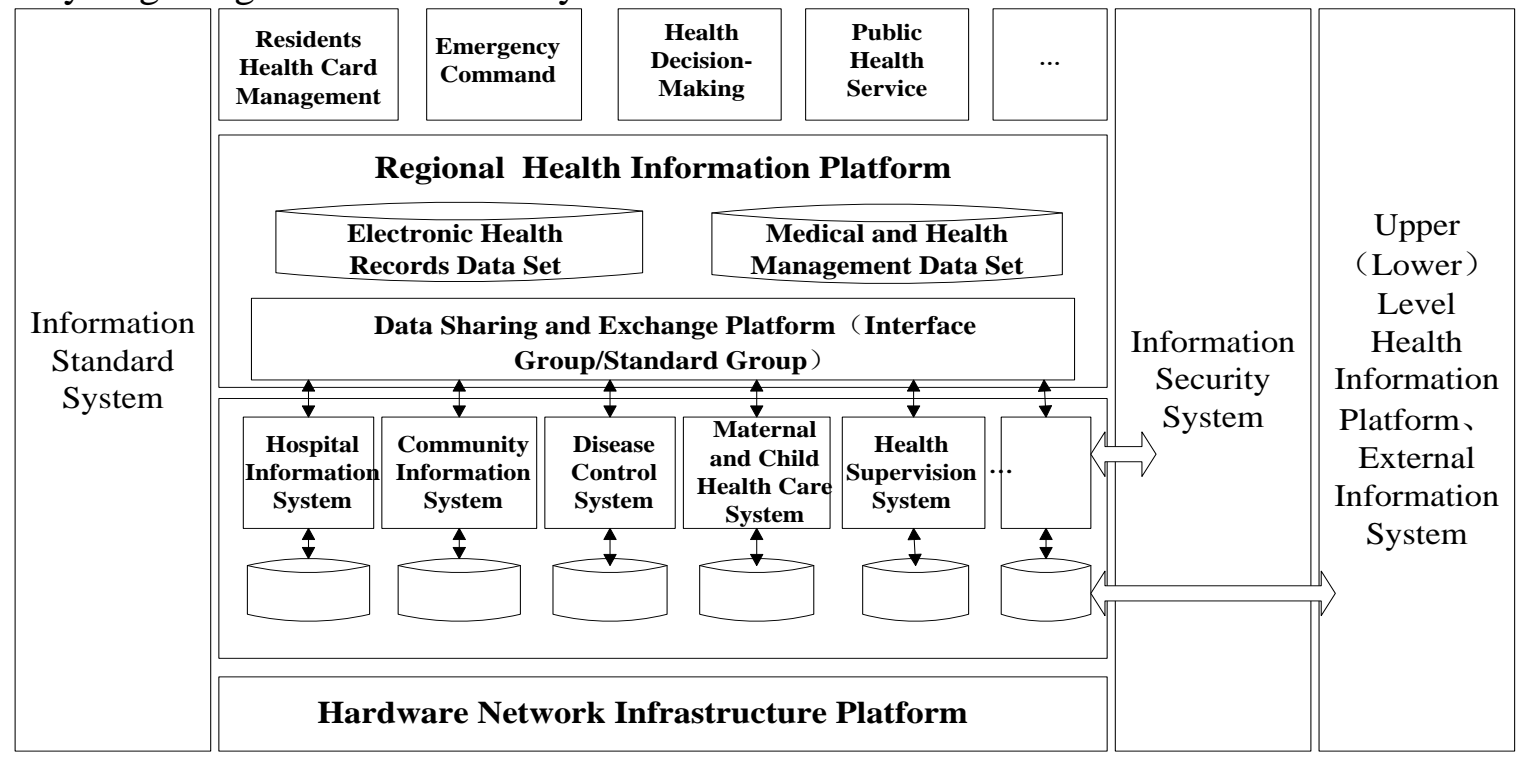

Fig. 1 Overall framework

Interaction process between the hospital and the community. The regional health information platform uses the resident health card as the only medium of treatment in all hospitals and 
communities. And the regional health information platform uses the identity card number as a unique identification of the resident's electronic health records. The regional health information platform has recorded the resident's electronic health records, including outpatient records in hospitals and communities, inpatient electronic medical records, inspection information etc. The communities and hospitals all upload and retrieve information in the regional health information system that is shown in Fig.2.The interaction process realizes the information sharing taking the health information of the residents as the center between different communities and hospitals.

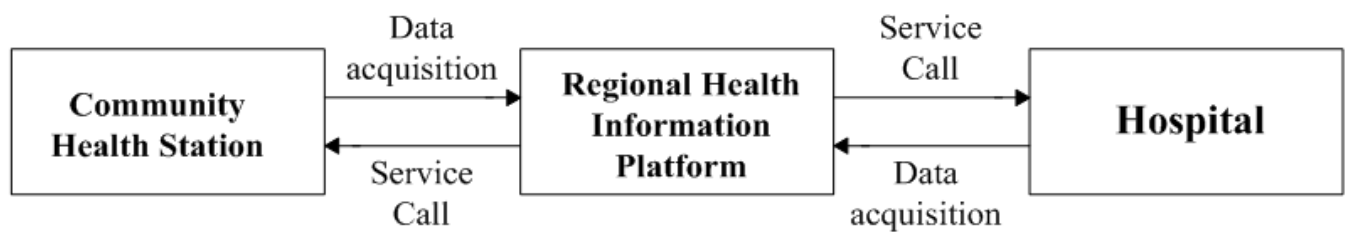

Fig. 2 Interaction process between the hospital and the community

\section{Research on Key Technology}

Information standardization. The related technology, standards, protocols and interfaces used by the regional information platform should follow relevant regulations of international, national and industry. The data structure and the design of the application software should implement the relevant standards of the industry [3]. First the regional health information platform should refer to regulations of the basic framework and data standard of the health records (for trial implementation) and regional health information platform construction guide based the health records (for trial implementation) issued by the Ministry of health. Secondly the construction of professional system should accord to corresponding industry standards, such as the use of DICOMM (Digital Imaging and Communications in Medicine) to realize the standardization of medical imaging and the use of HL7 (Health Level Seven) to realize the standardization of electronic medical records.

Data acquisition and access technology. Due to the large outpatient amount and the large volume of business the amount of data to interact in the hospital is also large. In order to ensure the normal operation of the hospital business system and the information sharing of the regional health information platform the pre-machine can be used in the hospital to complete the interaction with the regional health information platform. When the patient registers in the hospital the identity card number is uploaded to the pre-machine and the pre-machine downloads the electronic health information of the patient to the doctor for the use of treatment. In addition the pre-machine automatic extracts the medical and treatment information from the hospital systems and uploads to the regional health information platform.

The community health service stations are all small in scale, so the data acquisition and accessing can use directly way of uploading and accessing. If the system is not easy to directly upload, it can also use the mode of manual importing.

Network transmission technology. Regional health information platform involves all the hospitals and the community health service station in the region. The locations of the various medical institutions are scattered and some may be relatively remote. Therefore the network transmission can use a variety of combination. For the large hospitals that the locations are near and the conditions are ripe these can use dedicated line access. But the cost of the dedicated line access is higher and the flexibility is not good. For the medical institutions that the locations are remote and there are no dedicated lines those can use the access mode of VPN (Virtual Private Network) [4]. VPN has to access methods that are SSL (Secure Sockets Layer) VPN and IPsec (Internet Protocol Security) VPN according to the use of different protocols. The way of SSL VPN is more flexible and convenient and the cost is low. The way of IPsec VPN need to install equipment in the client and the realization is complicated.

Data storage technology. The regional health information platform stores the electronic health record information and the electronic medical record information. The data storage of the regional 
health information platform can use those ways including centralized, distributed and mixed ways [5]. The centralized storage is to build a unified data center to store all the data in the hospitals and communities such as the electronic health record information. Virtualization technology can be used in order to ensure the efficient management of the centralized data store and reduce the cost. The distributed storage is to store the data in each institution and save the indexed in the regional health information platform such as the medical image information of the residents. The mixed storage is the combined way of the centralized way and the distributed way.

Information security technology. The electronic health record information of the regional health information platform refers to the privacy of the residents. Once the electronic health record information is leaked that will seriously affects the social order. So the construction of the regional health information platform should be strictly in accordance with requirements of the national security level protection system to ensure the authenticity, integrity, confidentiality, availability, reliability and controllability of the information. The regional health information platform should have the ability of safety protection, the ability of discovering the hidden trouble and the ability of emergency response. Therefore it needs to deploy equipment's of safety isolation, intrusion prevention, malicious code defense and application layer firewall in front of the intranet hosts. The security of the services provided to external by the regional health information platform need further strengthen. By employing the new method of the application layer defenses the attacks of the WEB such as the SQL injection and cross site scripting to ensure the anti-leakage of the information and the tamper proof of the portal site.

\section{Acknowledgments}

The paper is supported by the hospital level project of south area of Guanganmen hospital Chinese academy of traditional Chinese medicine (project number: y2013-13). The corresponding author of the paper is Zhang Hong who is the director of the department of computer center in Guanganmen hospital Chinese academy of traditional Chinese medicine.

\section{Summary}

The paper proposes the information sharing mode based the regional health information platform combing with the current situation of the hospital and the community information system. With the help of the computer technology, network technology and information security technology the regional health information platform builds an efficient, rapid and smooth network system covering at all levels of the medical institutions. The information sharing between the hospital and the community based the regional health information platform realizes the comprehensive application of the medical service and makes doctors in the community conveniently and timely know the diagnosis and treatment in the hospital ,which establishes a division of labor and cooperation mechanism and improves the health service ability in the community,

\section{References}

[1] Hui Wang, Wei Liu, Shihong Zhang, J. Journal of Medical Informatics. 7 (2010) 14-17.

[2] Junhua Zhong, Baomei Liu, Jinxiong Chen, J. China Digital Medicine. 9(2013) 12-14.

[3] Bin Song, Haidong Chen, Chunyu Zheng, Saiyu J. Hosp Adm in J Chin PLA. 9 (2010)819-821.

[4] Jing Li, Hong Zhang, Xiang Li, J. China Digital Medicine. 5 (2014)102-104.

[5] Yu Tang, Jiazhi Liu, Huaping Gan, Wen Chen, Changqi Feng,Changqing Bu, J. Chinese Journal of Health Informatics and Management. 2 (2013)96-104,129. 